\title{
On the stability of a cubic functional equation in random 2-normed spaces
}

\author{
Abdullah Alotaibi and Syed Abdul Mohiuddine*
}

* Correspondence:

mohiuddine@gmail.com

Department of Mathematics,

Faculty of Science, King Abdulaziz University, P.O. Box 80203, Jeddah

21589, Saudi Arabia

\begin{abstract}
In this article, we propose to determine some stability results for the functional equation of cubic in random 2-normed spaces which seems to be a quite new and interesting idea. Also, we define the notion of continuity, approximately and conditional cubic mapping in random 2-normed spaces and prove some interesting results.
\end{abstract}

Keywords: distribution function, t-norm, triangle function, random 2-normed space, cubic functional equation, Hyers-Ulam-Rassias stability

\section{Introduction and preliminaries}

In 1940, Ulam [1] proposed the following question concerning the stability of group homomorphisms:

Let $G_{1}$ be a group and let $G_{2}$ be a metric group with the metric $d(.$, .). Given $\epsilon>0$, does there exist a $\delta>0$ such that if a function $h: G_{1} \rightarrow G_{2}$ satisfies the inequality $d(h$ $(x y), h(x) h(y))<\delta$ for all $x, y \in G_{1}$, then there exists a homomorphism $H: G_{1} \rightarrow G_{1}$ with $d(h(x), H(x))<\epsilon$ for all $x \in G_{1}$ ?

In next year, Hyers [2] answers the problem of Ulam under the assumption that the groups are Banach spaces and then generalized by Aoki [3] and Rassias [4] for additive mappings and linear mappings, respectively. Since then several stability problems for various functional equations have been investigated in [5-12].

The stability problem for the cubic functional equation was proved by Jun and Kim [5] for mappings $f: X \rightarrow Y$, where $X$ is a real normed space and $Y$ is a Banach space. Later on, the problem of stability of cubic functional equation were discussed by many mathematician.

An interesting and important generalization of the notion of a metric space was introduced by Menger [13] under the name of statistical metric space, which is now called a probabilistic metric space. An important family of probabilistic metric spaces is that of probabilistic normed spaces. The theory of probabilistic normed spaces is important as a generalization of deterministic results of linear normed spaces. The theory of probabilistic normed spaces was initiated and developed in $[14,15]$ and further it was extended to random 2-normed spaces by Goleț [16] using the concept of 2-norm of Gahler [17]. For more details of probabilistic and random/fuzzy 2-normed space, we refer to [18-22] and references therein.

(c) 2012 Alotaibi and Mohiuddine; licensee Springer. This is an Open Access article distributed under the terms of the Creative Commons Attribution License (http://creativecommons.org/licenses/by/2.0), which permits unrestricted use, distribution, and reproduction in any medium, provided the original work is properly cited. 
In this article, we establish Hyers-Ulam stability concerning the cubic functional equations in random 2-normed spaces which is quite a new and interesting idea to study with.

In this section, we recall some notations and basic definitions used in this article.

A distribution function is an element of $\Delta^{+}$, where $\Delta^{+}=\{f: \mathbb{R} \rightarrow[0,1] ; f$ is left-continuous, nondecreasing, $f(0)=0$ and $f(+\infty)=1\}$ and the subset $D^{+} \subseteq \Delta^{+}$is the set $D^{+}=$ $\left\{f \in \Delta^{+} ; l^{-} f(+\infty)=1\right\}$. Here $l f(+\infty)$ denotes the left limit of the function $f$ at the point $x$. The space $\Delta^{+}$is partially ordered by the usual point-wise ordering of functions, i.e., $f \leq$ $g$ if and only if $f(x) \leq g(x)$ for all $x \in \mathbb{R}$. For any $a \in \mathbb{R}, H_{a}$ is a distribution function defined by

$$
H_{a}(x)= \begin{cases}0 & \text { if } x \leq a \\ 1 & \text { if } x>a\end{cases}
$$

The set $\Delta$, as well as its subsets, can be partially ordered by the usual pointwise order: in this order, $H_{0}$ is the maximal element in $\Delta^{+}$.

A triangle function is a binary operation on $\Delta^{+}$, namely a function $\tau: \Delta^{+} \times \Delta^{+} \rightarrow \Delta^{+}$ that is associative, commutative nondecreasing and which has $\varepsilon_{0}$ as unit, that is, for all $f, g, h \in \Delta^{+}$, we have:

(i) $\tau(\tau(f, g), h)=\tau(f, \tau(g, h))$

(ii) $\tau(f, g)=\tau(g, f)$,

(iii) $\tau(f, g)=\tau(g, f)$ whenever $f \leq g$,

(iv) $\tau\left(f, H_{0}\right)=f$.

A $t$-norm is a continuous mapping *: $[0,1] \times[0,1] \rightarrow[0,1]$ such that $([0,1], *)$ is abelian monoid with unit one and $c * d \geq a * b$ if $c \geq a$ and $d \geq b$ for all $a, b, c, d \in$ $[0,1]$.

The concept of 2-normed space was first introduced in [17] and further studied in [23-25].

Let $\mathrm{X}$ is a linear space of a dimension $\mathrm{d}$, where $2 \leq \mathrm{d}<\infty$. A 2 -normed on $\mathrm{X}$ is a function $\|.,\|:. X \times X \rightarrow \mathbb{R}$ satisfying the following conditions, for every $\mathrm{x}, \mathrm{y} \in \mathrm{X}$ (i) $\|\mathrm{x}, \mathrm{y}\|=0$ if and only if $\mathrm{x}$ and $\mathrm{y}$ are linearly dependent; (ii) $\|\mathrm{x}, \mathrm{y}\|=\|\mathrm{y}, \mathrm{x}\|$; (iii) $\|\alpha \mathrm{x}, \mathrm{y}\|=|\alpha|\|\mathrm{x}, \mathrm{y}\|, \alpha \in \mathbb{R}$; (iv) $\| \mathrm{x}+$ $\mathrm{y}, \mathrm{z}\|\leq\| \mathrm{x}, \mathrm{z}\|+\| \mathrm{y}, \mathrm{z} \|$. In this case $(\mathrm{X},\|.,\|$.$) is called a 2-norm space.$

Example 1.1. Take $X=\mathbb{R}^{2}$ being equipped with the 2-norm $\|x, y\|=$ the area of the parallelogram spanned by the vectors $x$ and $y$, which may be given explicitly by the formula

$$
\|x, y\|=\left|x_{1} y_{2}-x_{2} y_{1}\right|, \text { where } x=\left(x_{1}, x_{2}\right), y=\left(y_{1}, y_{2}\right) .
$$

Recently, Goleț [16] introduced the notion of random 2-normed space and further studied by Mursaleen [26].

Let $X$ be a linear space of a dimension greater than one, $\tau$ is a triangle function, and $\mathcal{F}: X \times X \rightarrow \Delta^{+}$. Then $\mathscr{\mathscr { F }}$ is called a probabilistic 2-norm on $X$ and $(X, \mathcal{F}, \tau)$ a probabilistic 2-normed space if the following conditions are satisfied:

(i) $\mathcal{F}_{x, y}(t)=H_{0}(t)$ if $x$ and $y$ are linearly dependent, where $\mathcal{F}_{x, y}(t)$ denotes the value of $\mathcal{F}_{x, y}$ at $t \in \mathbb{R}$,

(ii) $\mathcal{F}_{x, y} \neq H_{0}$ if $x$ and $y$ are linearly independent, 
(iii) $\mathcal{F}_{x, y}=\mathcal{F}_{y, x}$ for every $x, y$ in $X$,

(iv) $\mathcal{F}_{\alpha x, y}(t)=\mathcal{F}_{x, y}\left(\frac{t}{|\alpha|}\right)$ for every $t>0, \alpha \neq 0$ and $x, y \in X$,

(v) $\mathcal{F}_{x+y, z} \geq \tau\left(\mathcal{F}_{x, z}, \mathcal{F}_{y, z}\right)$ whenever $x, y, z \in X$.

If $(\mathrm{v})$ is replaced by

(v') $\mathcal{F}_{x+y, z}\left(t_{1}+t_{2}\right) \geq \mathcal{F}_{x, z}\left(t_{1}\right) * \mathcal{F}_{y, z}\left(t_{2}\right)$, for all $x, y, z \in X$ and $t_{1}, t_{2} \in \mathbb{R}_{0}^{+}$, then triple $(X, \mathcal{F}, *)$ is called a random 2-normed space (for short, RTN-space).

Example 1.2. Let $(X,\|.\|$,$) be a 2$-normed space with $\|x, z\|=\left\|x_{1} z_{2}-x_{2} z_{1}\right\|, x=\left(x_{1}\right.$, $\left.x_{2}\right), z=\left(z_{1}, z_{2}\right)$ and $a * b=a b$ for $a, b \in[0,1]$. For all $x \in X, t>0$ and nonzero $z \in$ $X$, consider

$$
\mathcal{F}_{x, z}(t)= \begin{cases}\frac{t}{t+\|x, z\|} & \text { if } t>0 \\ 0 & \text { if } t \leq 0\end{cases}
$$

Then $(X, \mathcal{F}, *)$ is a random 2-normed space.

Remark 1.3. Note that every 2-normed space $(X,\|.\|$,$) can be made a random 2-$ normed space in a natural way, by setting $\mathcal{F}_{x, y}(t)=H_{0}(t-\|x, y\|)$, for every $x, y \in X$, $t>0$ and $a * b=\min \{a, b\}, a, b \in[0,1]$.

\section{Stability of cubic functional equation}

In the present section, we define the notion of convergence, Cauchy sequence and completeness in RTN-space and determine some stability results of the cubic functional equation in RTN-space.

The functional equation

$$
f(2 x+y)+f(2 x-y)=2 f(x+y)+2 f(x-y)+12 f(x)
$$

is called the cubic functional equation, since the function $f(x)=c x^{3}$ is its solution. Every solution of the cubic functional equation is said to be a cubic mapping.

We shall assume throughout this article that $X$ and $Y$ are linear spaces; $(X, \mathcal{F}, *)$ and $\left(Z, \mathcal{F}^{\prime}, *\right)$ are random 2-normed spaces; and $(Y, \mathcal{F}, *)$ is a random 2-Banach space.

Let $\phi$ be a function from $X \times X$ to $Z$. A mapping $f: X \rightarrow Y$ is said to be $\phi$-approximately cubic function if

$$
\mathcal{F}_{E_{x, y}, z}(t) \geq \mathcal{F}_{\varphi(x, y), z}^{\prime}(t)
$$

for all $x, y \in X, t>0$ and nonzero $z \in X$, where

$$
E_{x, y}=f(2 x+y)+f(2 x-y)-2 f(x+y)-2 f(x-y)-12 f(x) .
$$

We define:

We say that a sequence $x=\left(x_{k}\right)$ is convergent in $(X, \mathcal{F}, *)$ or simply $\mathscr{F}$-convergent to $\ell$ if for every $\epsilon>0$ and $\theta \in(0,1)$ there exists $k_{0} \in \mathbb{N}$ such that $\mathcal{F}_{x_{k}-\ell, z}(\varepsilon)>1-\theta$ whenever $k \geq k_{0}$ and nonzero $z \in X$. In this case we write $\mathcal{F}-\lim _{k \rightarrow \infty} x_{k}=\ell$ and $\ell$ is called the $\mathscr{F}$-limit of $x=\left(x_{k}\right)$.

A sequence $x=\left(x_{k}\right)$ is said to be Cauchy sequence in $(X, \mathcal{F}, *)$ or simply $\mathscr{F}$-Cauchy if for every $\epsilon>0, \theta>0$ and nonzero $z \in X$ there exists a number $N=N(\epsilon, z)$ such that $\lim \mathcal{F}_{x_{n}-x_{m}, z}(\varepsilon)>1-\theta$ for all $n, m \geq N$. RTN-space $(X, \mathcal{F}, *)$ is said to be complete if 
every $\mathscr{F}$-Cauchy is $\mathscr{F}$-convergent. In this case $(X, \mathcal{F}, *)$ is called random 2 -Banach space.

Theorem 2.1. Suppose that a function $\phi: X \times X \rightarrow Z$ satisfies $\phi(2 x, 2 y)=\alpha \phi(x, y)$ for all $x, y \in X$ and $\alpha \neq 0$. Let $f: X \rightarrow Y$ be a $\phi$-approximately cubic function. If for some $0<\alpha<8$,

$$
\mathcal{F}_{\varphi(2 x, 2 y), z}^{\prime}(t) \geq \mathcal{F}_{\alpha \varphi(x, y), z}^{\prime}(t)
$$

and $\lim _{n \rightarrow \infty} \mathcal{F}_{\varphi\left(2^{n} x, 2^{n} y\right), z}^{\prime}\left(8^{n} t\right)=1$ for all $x, y \in X, t>0$ and nonzero $z \in X$. Then there exists a unique cubic mapping $C: X \rightarrow Y$ such that

$$
\mathcal{F}_{C(x)-f(x), z}(t) \geq \mathcal{F}_{\varphi(x, 0), z}^{\prime}((8-\alpha) t)
$$

for all $x \in X, t>0$ and nonzero $z \in X$.

Proof. For convenience, let us fix $y=0$ in (2). Then for all $x \in X, t>0$ and nonzero $z \in X$

$$
\mathcal{F}_{\frac{f(2 x)}{8}-f(x), z}\left(\frac{t}{16}\right) \geq \mathcal{F}_{\varphi(x, 0), z}^{\prime}(t) .
$$

Replacing $x$ by $2^{n} x$ in (5) and using (3), we obtain

$$
\mathcal{F}_{\frac{f\left(2^{n+1} x\right)}{8^{n+1}}-\frac{f\left(2^{n} x\right)}{8^{n}}, z}\left(\frac{t}{16\left(8^{n}\right)}\right) \geq \mathcal{F}_{\varphi\left(2^{n} x, 0\right), z}^{\prime}(t) \geq \mathcal{F}_{\varphi(x, 0), z}^{\prime}\left(t / \alpha^{n}\right),
$$

for all $x \in X, t>0$ and nonzero $z \in X$; and for all $n \geq 0$. By replacing $t$ by $\alpha^{n} t$, we get

$$
\mathcal{F}_{\frac{f\left(2^{n+1} x\right)}{8^{n+1}}-\frac{f\left(2^{n} x\right)}{8^{n}}, z}\left(\frac{\alpha^{n} t}{16\left(8^{n}\right)}\right) \geq \mathcal{F}_{\varphi(x, 0), z}^{\prime}(t)
$$

It follows from $\frac{f\left(2^{n} x\right)}{8^{n}}-f(x)=\sum_{k=0}^{n=1}\left(\frac{f\left(2^{k+1} x\right)}{8^{k+1}}-\frac{f\left(2^{k} x\right)}{8^{k}}\right)$ and (6) that

$$
\mathcal{F}_{\frac{f\left(2^{n} x\right)}{8^{n}}-f(x), z}\left(\sum_{k=0}^{n-1} \frac{\alpha^{k} t}{16\left(8^{k}\right)}\right) \geq \prod_{k=0}^{n-1} \mathcal{F}_{\frac{f\left(2^{k+1} x\right)}{8^{k+1}}-\frac{f\left(2^{k} x\right)}{8^{k}}, z}\left(\frac{\alpha^{k} t}{16\left(8^{k}\right)}\right) \geq \mathcal{F}_{\varphi(x, 0), z}^{\prime}(t),
$$

for all $x \in X, t>0$ and $n>0$ where $\prod_{j=1}^{n} a_{j}=a_{1} * a_{2} * \cdots * a_{n}$. By replacing $x$ with $2^{m} x$ in (7), we have

$$
\mathcal{F}_{\frac{f\left(2^{n+m} x\right)}{8^{n+m}}-\frac{f\left(2^{m} x\right)}{8^{m}}, z}\left(\sum_{k=0}^{n-1} \frac{\alpha^{k} t}{16(8)^{k+m}}\right) \geq \mathcal{F}_{\varphi\left(2^{m} x, 0\right), z}^{\prime}(t) \geq \mathcal{F}_{\varphi(x, 0), z}^{\prime}\left(t / \alpha^{m}\right) .
$$

Thus

$$
\mathcal{F}_{\frac{f\left(2^{n+m} x\right)}{8^{n+m}}-\frac{f\left(2^{m} x\right)}{8^{m}}, z}\left(\sum_{k=m}^{n+m-1} \frac{\alpha^{k} t}{16(8)^{k}}\right) \geq \mathcal{F}_{\varphi(x, 0), z}^{\prime}(t)
$$


for all $x \in X, t>0, m>0, n \geq 0$ and nonzero $z \in X$. Hence

$$
\mathcal{F}_{\frac{f\left(2^{n+m} x\right)}{8^{n+m}}-\frac{f\left(2^{m} x\right)}{8^{m}}, z}(t) \geq \mathcal{F}_{\varphi(x, 0), z}^{\prime}\left(\frac{t}{\sum_{k=m}^{n+m-1} \frac{\alpha^{k}}{16(8)^{k}}}\right),
$$

for all $x \in X, t>0 m \geq 0, n \geq 0$ and nonzero $z \in X$. Since $0<\alpha<8$ and $\sum_{k=0}^{\infty}\left(\frac{\alpha}{8}\right)^{k}<\infty$, the Cauchy criterion for convergence shows that $\left(\frac{f\left(2^{n} x\right)}{8^{n}}\right)$ is a Cauchy sequence in $(Y, \mathcal{F}, *)$. Since $(Y, \mathcal{F}, *)$ is complete, this sequence converges to some point $C(x) \in Y$. Fix $x \in X$ and put $m=0$ in (8) to obtain

$$
\mathcal{F}_{\frac{f\left(2^{n} x\right)}{8^{n}}-f(x), z}(t) \geq \mathcal{F}_{\varphi(x, 0), z}^{\prime}\left(\frac{t}{\sum_{k=0}^{n-1} \frac{\alpha^{k}}{16(8)^{k}}}\right),
$$

for all $t>0, n>0$ and nonzero $z \in X$. Thus we obtain

$$
\mathcal{F}_{C(x)-f(x), z}(t) \geq \mathcal{F}_{C(x)-\frac{f\left(2^{n} x\right)}{8^{n}}, z}(t / 2) * \mathcal{F}_{\frac{f\left(2^{n} x\right)}{8^{n}}-f(x), z}(t / 2) \geq \mathcal{F}_{\varphi(x, 0), z}^{\prime}\left(\frac{t}{\sum_{k=0}^{n-1} \frac{a^{k}}{8(8)^{k}}}\right),
$$

for large $n$. Taking the limit as $n \rightarrow \infty$ and using the definition of RTN-space, we get

$$
\mathcal{F}_{C(x)-f(x), z}(t) \geq \mathcal{F}_{\varphi(x, 0), z}^{\prime}((8-\alpha) t) .
$$

Replace $x$ and $y$ by $2^{n} x$ and $2^{n} y$, respectively, in (2), we have

$$
\mathcal{F}_{\frac{E_{2} n_{x, 2} n_{y}}{8^{n}}, z}(t) \geq \mathcal{F}_{\varphi\left(2^{n} x, 2^{n} y\right), z}^{\prime}\left(8^{n} t\right)
$$

for all $x, y \in X, t>0$ and nonzero $z \in X$. Since

$$
\lim _{n \rightarrow \infty} \mathcal{F}_{\varphi\left(2^{n} x, 2^{n} y\right), z}^{\prime}\left(8^{n} t\right)=1,
$$

we observe that $C$ fulfills (1). To Prove the uniqueness of the cubic function $C$, assume that there exists a cubic function $D: X \rightarrow Y$ which satisfies (4). For fix $x \in X$, clearly $C\left(2^{n} x\right)=8^{n} C(x)$ and $D\left(2^{n} x\right)=8^{n} D(x)$ for all $n \in \mathbb{N}$. It follows from (4) that

$$
\begin{aligned}
\mathcal{F}_{C(x)-D(x), z}(t) & =\mathcal{F}_{\frac{C\left(2^{n} x\right)}{8^{n}}-\frac{D\left(2^{n} x\right)}{8^{n}}, z}(t) \geq \mathcal{F}_{\frac{C\left(2^{n} x\right)}{8^{n}}-\frac{f\left(2^{n} x\right)}{8^{n}}, z}\left(\frac{t}{2}\right) * \mathcal{F}_{\frac{f\left(2^{n} x\right)}{8^{n}}-\frac{D\left(2^{n} x\right)}{8^{n}}, z}\left(\frac{t}{2}\right) \\
& \geq \mathcal{F}_{\varphi\left(2^{n} x, 0\right), z}^{\prime}\left(\frac{8^{n}(8-\alpha) t}{2}\right) \geq \mathcal{F}_{\varphi(x, 0), z}^{\prime}\left(\frac{8^{n}(8-\alpha) t}{2 \alpha^{n}}\right) .
\end{aligned}
$$

Therefore

$$
\mathcal{F}_{\varphi(x, 0), z}^{\prime}\left(\frac{8^{n}(8-\alpha) t}{2 \alpha^{n}}\right)=1 .
$$

Thus $\mathcal{F}_{C(x)-D(x), z}(t)=1$ for all $x \in X, t>0$ and nonzero $z \in X$. Hence $C(x)=D(x)$.

Example 2.2. Let $X$ be a Hilbert space and $Z$ be a normed space. By $\mathscr{F}$ and $\mathcal{F}^{\prime}$, we denote the random 2-norms given as in Example 1.1 on $\mathrm{X}$ and $\mathrm{Z}$, respectively. Let $\phi$ : $X \times X \rightarrow Z$ be defined by $\phi(x, y)=8\left(\|x\|^{2}+\|y\|^{2}\right) z_{\mathrm{o}}$, where $z_{\mathrm{o}}$ is a fixed unit vector in $Z$. Define $f: X \rightarrow X$ by $f(x)=\|x\|^{2} x+\|x\|^{2} x_{\mathrm{o}}$ for some unit vector $x_{\mathrm{o}} \in X$. Then 


$$
\mathcal{F}_{E_{x, y}, z}(t)=\frac{t}{t+8\|x, z\|^{2}+2\|y, z\|^{2}} \geq \frac{t}{t+8\|x, z\|^{2}+8\|y, z\|^{2}}=\mathcal{F}_{\varphi(x, y), z}^{\prime}(t)
$$

Also

$$
\mathcal{F}_{\varphi(2 x, 0), z}^{\prime}(t)=\frac{t}{t+32\|x, z\|^{2}}=\mathcal{F}_{4 \varphi(x, 0), z}^{\prime}(t)
$$

Thus,

$$
\lim _{n \rightarrow \infty} \mathcal{F}_{\varphi\left(2^{n} x, 2^{n} y\right), z}^{\prime}\left(8^{n} t\right)=\lim _{n \rightarrow \infty} \frac{8^{n} t}{8^{n} t+8\left(4^{n}\right)\left(\|x, z\|^{2}+\|y, z\|^{2}\right)}=1 .
$$

Hence, conditions of Theorem 2.1 for $\alpha=4$ are fulfilled. Therefore, there is a unique cubic mapping $C: X \rightarrow X$ such that $\mathcal{F}_{C(x)-f(x), z}(t) \geq \mathcal{F}_{\varphi(x, 0), z}^{\prime}(4 t)$ for all $x \in X, t>0$ and nonzero $z \in X$.

By a modification in the proof of Theorem 2.1, one can easily prove the following:

Theorem 2.3. Suppose that a function $\phi: X \times X \rightarrow Z$ satisfies $\varphi(x / 2, y / 2)=\frac{1}{\alpha} \varphi(x, y)$ for all $x, y \in X$ and $\alpha \neq 0$. Let $f: X \rightarrow Y$ be a $\phi$-approximately cubic function. If for some $\alpha>8$

$$
\mathcal{F}_{\varphi(x / 2, y / 2), z}^{\prime}(t) \geq \mathcal{F}_{\varphi(x, y), z}^{\prime}(\alpha t)
$$

and $\lim _{n \rightarrow \infty} \mathcal{F}_{8^{n} \varphi\left(2^{-n} x, 2^{-n} y\right), z}^{\prime}(t)=1$ for all $x, y \in X, t>0$ and nonzero $z \in X$. Then there exists a unique cubic mapping $C: X \rightarrow Y$ such that

$$
\mathcal{F}_{C(x)-f(x), z}(t) \geq \mathcal{F}_{\varphi(x, 0), z}^{\prime}((\alpha-8) t),
$$

for all $x \in X, t>0$ and nonzero $z \in X$.

\section{Continuity in random 2-normed spaces}

In this section, we establish some interesting results of continuous approximately cubic mappings.

Let $f: \mathbb{R} \rightarrow X$ be a function, where $\mathbb{R}$ is endowed with the Euclidean topology and $X$ is an random 2-normed space equipped with random 2-norm $\mathscr{\mathscr { H }}$. Then, $f$ is said to be random 2 -continuous or simply $\mathscr{F}$-continuous at a point $s_{\mathrm{o}} \in \mathbb{R}$ if for all $\epsilon>0$ and all 0 $<\alpha<1$ there exists $\delta>0$ such that

$$
\mathcal{F}_{f(s x)-f\left(s_{\circ} x\right), z}(\varepsilon) \geq \alpha,
$$

for each $s$ with $0<\left|s-s_{\mathrm{o}}\right|<\delta$ and nonzero $z \in X$.

A mapping $f: X \rightarrow Y$ is said to be $(p, q)$-approximately cubic function if, for some $p$, $q$ and some $z_{\mathrm{o}} \in Z$,

$$
\mathcal{F}_{E_{x, y}, z}(t) \geq \mathcal{F}_{\left(\|x\|^{p}+\|y\|^{q}\right) z_{0}, z}^{\prime}(t),
$$

for all $x, y \in X, t>0$ and nonzero $z \in X$.

Theorem 3.2. Let $X$ be a normed space and let $f: X \rightarrow Y$ be a $(p, q)$-approximately cubic function. If $p, q<3$, there exists a unique cubic mapping $C: X \rightarrow Y$ such that 


$$
\mathcal{F}_{C(x)-f(x), z}(t) \geq \mathcal{F}_{\|x\|^{p} z_{0}, z}^{\prime}\left(\left(8-2^{p}\right) t\right),
$$

for all $x \in X, t>0$ and nonzero $z \in X$. Furthermore, if for some $x \in X$ and all $n \in$ $\mathbb{N}$, the mapping $g: \mathbb{R} \rightarrow Y$ defined by $g(s)=f\left(2^{n} s x\right)$ is $\mathscr{F}$-continuous. Then the mapping $s \mapsto C(s x)$ from $\mathbb{R}$ to $Y$ is $\mathscr{F}$-continuous; in this case, $C(r x)=r^{3} C(x)$ for all $r \in \mathbb{R}$.

Proof. Suppose that a function $\phi: X \times X \rightarrow Z$ satisfies $\phi(x, y)=\left(\|x\|^{p}+\|y\|^{q}\right) z_{\mathrm{o}}$. Existence and uniqueness of the cubic mapping $C$ satisfying (9) are deduced from Theorem 2.1. Note that for each $x \in X, t \in \mathbb{R}$ and $n \in \mathbb{N}$, we have

$$
\mathcal{F}_{C(x)-\frac{f\left(2^{n} x\right)}{8^{n}, z}}(t)=\mathcal{F}_{C\left(2^{n} x\right)-f\left(2^{n} x\right), z}\left(8^{n} t\right) \geq \mathcal{F}_{2^{n p}\|x\| \|^{2} z_{0}, z}^{\prime}\left(8^{n}\left(8-2^{p}\right) t\right)=\mathcal{F}_{\|x\| \|_{0} z_{0}, z}^{\prime}\left(\frac{8^{n}\left(8-2^{p}\right) t}{2^{n p}}\right) .
$$

Fix $x \in X$ and $s_{\mathrm{o}} \in \mathbb{R}$. Given $\epsilon>0$ and $0<\alpha<1$. From (10) follows that

$$
\mathcal{F}_{C(s x)-\frac{f\left(2^{n} s x\right)}{8^{n}}, z}(t) \geq \mathcal{F}_{\|x\|^{p} z_{o}, z}^{\prime}\left(\frac{8^{n}\left(8-2^{p}\right) t}{|s|^{p} 2^{n p}}\right) \geq \mathcal{F}_{\|x\|^{p} z_{o}, z}^{\prime}\left(\frac{8^{n}\left(8-2^{p}\right) t}{\left(1+\left|s_{\circ}\right|\right)^{p} 2^{n p}}\right),
$$

for all $\left|s-s_{\mathrm{o}}\right|<1$ and $s \in \mathbb{R}$. Since $\lim _{n \rightarrow \infty} \frac{8^{n}\left(8-2^{p}\right) t}{\left(1+\left|s_{0}\right|\right)^{p} 2^{n p}}=\infty$, there exists $n_{\mathrm{o}} \in \mathbb{N}$ such that

$$
\mathcal{F}_{C(s x)-\frac{f\left(2^{n_{0}} s x\right)}{8^{n_{\circ}}}, z}\left(\frac{\varepsilon}{3}\right) \geq \alpha,
$$

for all $\left|s-s_{\mathrm{o}}\right|<1$ and $s \in \mathbb{R}$. By the $\mathscr{F}$-continuity of the mapping $t \rightarrow f\left(2^{n_{\circ}} t x\right)$, there exists $\delta<1$ such that for each $s$ with $0<\left|s-s_{\mathrm{o}}\right|<\delta$, we have

$$
\mathcal{F}_{\frac{f\left(2^{n_{\circ}} s x\right)}{8^{n_{o}}}-\frac{f\left(2^{n_{o}} s_{o} x\right)}{8^{n_{\circ}}}, z}\left(\frac{\varepsilon}{3}\right) \geq \alpha .
$$

It follows that

$$
\begin{aligned}
& \mathcal{F}_{C(s x)-C\left(s_{0} x\right), z}(\varepsilon) \\
& \quad \geq \mathcal{F}_{C(s x)-\frac{f\left(2^{n_{o}} x\right)}{8^{n_{o}}}, z}\left(\frac{\varepsilon}{3}\right) * \mathcal{F}_{\frac{f\left(2^{n_{o}} s x\right)}{8^{n_{o}}}-\frac{f\left(2^{n_{o}} s_{s} x\right)}{8^{o_{o}}}, z}\left(\frac{\varepsilon}{3}\right) * \mathcal{F}_{C\left(s_{o} x\right)-\frac{f\left(2^{n_{o}} s_{x} x\right)}{8^{n_{o}}}, z}\left(\frac{\varepsilon}{3}\right) \geq \alpha,
\end{aligned}
$$

for each $s$ with $0<\left|s-s_{\mathrm{o}}\right|<\delta$. Hence, the mapping $s \mapsto C(s x)$ is $\mathscr{F}$-continuous.

Now, we use the $\mathscr{F}$-continuity of $s \mapsto C(s x)$ to establish that $C\left(r_{\circ} x\right)=r_{\circ}^{3} C(x)$ for all $r_{\mathrm{o}}$ $\in \mathbb{R}$. For each $r, \mathbb{Q}$ is a dense subset of $\mathbb{R}$, we have $C(r x)=r^{3} C(x)$. Fix $r_{\mathrm{o}} \in \mathbb{R}$ and $t>0$. Then, for $0<\alpha<1$ there exists $\delta>0$ such that

$$
\mathcal{F}_{C(r x)-C\left(r_{\circ} x\right), z}(t / 3) \geq \alpha,
$$

for each $r \in \mathbb{R}$ and $0<\left|r-r_{\mathrm{o}}\right|<\delta$. Choose a rational number $r$ with $0<\left|r-r_{\mathrm{o}}\right|<\delta$ and $\left|r^{3}-r_{\circ}^{3}\right|<1-\alpha$. Then

$$
\begin{gathered}
\mathcal{F}_{C\left(r_{0} x\right)-r_{0}^{3} C(x), z}(t) \geq \mathcal{F}_{C\left(r_{\circ} x\right)-C(r x), z}(t / 3) * \mathcal{F}_{C(r x)-r^{3} C(x), z}(t / 3) * \mathcal{F}_{r^{3} C(x)-r_{0}^{3} C(x), z}(t / 3) \\
\geq \alpha * 1 * \mathcal{F}_{C(x), z}(t / 3(1-\alpha)) .
\end{gathered}
$$

Thus $\mathcal{F}_{C\left(r_{\circ} x\right)-r_{0}^{3} C(x), z}(t)=1$. Hence, we conclude that $C\left(r_{\circ} x\right)=r_{\circ}^{3} C(x)$.

Remark 3.2. We can also prove Theorem 3.1 for the case when $p, q>3$. In this case, there exists a unique cubic mapping $C: X \rightarrow Y$ such that $\mathcal{F}_{C(x)-f(x), z}(t) \geq \mathcal{F}_{\|x\|^{p} z_{0}, z}^{\prime}\left(\left(2^{p}-8\right) t\right)$ for all $x \in X, t>0$ and nonzero $z \in X$. 
4 Approximately and conditional cubic mapping in random 2-normed spaces In this section, we obtain completeness in RTN-space through the existence of some solution of a stability problem for cubic functional equation.

A mapping $f: \mathbb{N u}\{0\} \rightarrow X$ is said to be approximately cubic if for each $\alpha \in(0,1)$ there exists some $n_{\alpha} \in \mathbb{N}$ such that $\mathcal{F}_{E(n, m), z}(1) \geq \alpha$, for all $n \geq 2 m \geq n_{\alpha}$ and nonzero $z \in X$.

By a conditional cubic mapping, we mean a mapping $f: \mathbb{N} \cup\{0\} \rightarrow X$ such that (1) holds whenever $x \geq 2 y$.

It can be easily verified that for each conditional cubic mapping $f: \mathbb{N} \cup\{0\} \rightarrow X$, we have $f\left(2^{n}\right)=2^{3 n} f(1)$.

Theorem 4.1. Let $(X, \mathcal{F}, *)$ be a RTN-space such that for each approximately cubic mapping $f: \mathbb{N} \cup\{0\} \rightarrow X$, there exists a conditional cubic mapping $C: \mathbb{N} \cup\{0\} \rightarrow X$, such that

$$
\lim _{n \rightarrow \infty} \mathcal{F}_{C(n)-f(n), z}(1)=1,
$$

for nonzero $z \in X$. Then $(X, \mathcal{F}, *)$ is a random 2-Banach space.

Proof. Let $\left(x_{n}\right)$ be a Cauchy sequence in a RTN-space. By induction on $k$, we can find a strictly increasing sequence $\left(n_{k}\right)$ of natural numbers such that

$$
\mathcal{F}_{x_{n}-x_{m}, z}\left(\frac{1}{(10 k)^{3}}\right) \geq 1-\frac{1}{k}
$$

for each $n, m \geq n_{k}$ and nonzero $z \in X$. Let $y_{k}=x_{n_{k}}$ and define $f: \mathbb{N} \cup\{0\} \rightarrow X$ by $f(k)$ $=k^{3} y_{k}$. Let $\alpha \in(0,1)$. and find some $n_{\mathrm{o}} \in \mathbb{N}$ such that $1-\frac{1}{n_{\circ}}>\alpha$. One can easily verified that

$$
\begin{aligned}
& \mathcal{F}_{E_{k, j}, z}(1) \geq \mathcal{F}_{\gamma_{2 k+j}-\gamma_{k+j}, z}\left(\frac{1}{20 k^{3}}\right) * \mathcal{F}_{\gamma_{2 k+j}-\gamma_{k-j}, z}\left(\frac{1}{20 k^{3}}\right) * \mathcal{F}_{\gamma_{2 k+j}-\gamma_{k}, z}\left(\frac{1}{40 k^{3}}\right) \\
& * \mathcal{F}_{\gamma_{2 k-j}-\gamma k, z}\left(\frac{1}{80 k^{3}}\right) * \mathcal{F}_{\gamma_{2 k+j}-\gamma_{2 k-j, z}}\left(\frac{1}{120 k^{2} j}\right) * \mathcal{F}_{\gamma_{k-j}-\gamma_{k+j}, z}\left(\frac{1}{60 k^{2} j}\right) \\
& * \mathcal{F}_{\gamma_{2 k+j}-\gamma_{k+j}, z}\left(\frac{1}{60 k j^{2}}\right) * \mathcal{F}_{\gamma_{2 k-j}-y_{k-j,}, z}\left(\frac{1}{60 k j^{2}}\right) \\
& * \mathcal{F}_{\gamma_{2 k+j}-\gamma_{2 k-j, z}}\left(\frac{1}{10 j^{3}}\right) * \mathcal{F}_{\gamma_{k-j}-y_{k+j}, z}\left(\frac{1}{20 j^{3}}\right) \text {, }
\end{aligned}
$$

for each $k \geq 2 j$, and nonzero $z \in X$. Then

$$
\mathcal{F}_{\gamma_{2 k+j}-\gamma_{k+j}, z}\left(\frac{1}{20 k^{3}}\right) \geq \mathcal{F}_{\gamma_{2 k+j}-\gamma_{k+j}, z}\left(\frac{1}{20(k+j)^{3}}\right) \geq \mathcal{F}_{\gamma_{2 k+j}-\gamma_{k+j}, z}\left(\frac{1}{10^{3}(k+j)^{3}}\right) \geq \alpha,
$$


for $j>n_{\mathrm{o}}$ and nonzero $z \in X$. Since $k-j \geq \frac{k}{2}$ and $k-j \geq j$, we have

$$
\begin{aligned}
\mathcal{F}_{\gamma_{2 k+j}-\gamma_{k-j}, z}\left(\frac{1}{20 k^{3}}\right) & \geq \mathcal{F}_{\gamma_{2 k+j}-y_{k-j, z}}\left(\frac{1}{10^{3}(k-j)^{3}}\right) \geq \alpha, \\
\mathcal{F}_{\gamma_{k-j}-\gamma_{k+j}, z}\left(\frac{1}{60 k^{2} j}\right) & \geq \mathcal{F}_{\gamma_{k-j}-y_{k+j,}, z}\left(\frac{1}{10^{3}(k-j)^{3}}\right) \geq \alpha, \\
\mathcal{F}_{\gamma_{2 k-j}-\gamma_{k-j,}, z}\left(\frac{1}{60 k j^{2}}\right) & \geq \mathcal{F}_{\gamma_{2 k-j}-y_{k-j}, z}\left(\frac{1}{10^{3}(k-j)^{3}}\right) \geq \alpha, \\
\mathcal{F}_{\gamma_{k-j}-\gamma_{k+j,}, z}\left(\frac{1}{20 j^{3}}\right) & \geq \mathcal{F}_{\gamma_{2 k-j}-\gamma_{k-j}, z}\left(\frac{1}{10^{3}(k-j)^{3}}\right) \geq \alpha .
\end{aligned}
$$

Clearly

$$
\begin{aligned}
& \mathcal{F}_{\gamma_{2 k+j}-\gamma_{k}, z}\left(\frac{1}{40 k^{3}}\right) \geq \mathcal{F}_{y_{2 k+j}-\gamma_{k, z}}\left(\frac{1}{10^{3} k^{3}}\right) \geq \alpha, \mathcal{F}_{y_{2 k-j}-\gamma_{k, z}, z}\left(\frac{1}{80 k^{3}}\right) \geq \mathcal{F}_{\gamma_{2 k-j}-\gamma_{k}, z}\left(\frac{1}{10^{3} k^{3}}\right) \geq \alpha, \\
& \text { and } \mathcal{F}_{\gamma_{2 k+j}-\gamma_{k+j, z}}\left(\frac{1}{60 k j^{2}}\right) \geq \mathcal{F}_{y_{2 k+j}-\gamma_{k+j}, z}\left(\frac{1}{10^{3}(k+j)^{3}}\right) \geq \alpha \text {. }
\end{aligned}
$$

The inequalities $2 k-j \geq j$ and $2 k-j>k$ imply

$$
\begin{aligned}
\mathcal{F}_{\gamma_{2 k+j}-\gamma_{2 k-j, z}}\left(\frac{1}{120 k^{2} j}\right) & \geq \mathcal{F}_{\gamma_{2 k+j}-\gamma_{2 k-j, z}}\left(\frac{1}{120(2 k-j)^{3}}\right) \geq \mathcal{F}_{y_{2 k+j}-\gamma_{2 k-j, z}}\left(\frac{1}{10^{3}(2 k-j)^{3}}\right) \geq \alpha \\
\mathcal{F}_{\gamma_{2 k+j}-\gamma_{2 k-j, z}}\left(\frac{1}{10 j^{3}}\right) & \geq \mathcal{F}_{\gamma_{2 k+j}-\gamma_{2 k-j, z}}\left(\frac{1}{10(2 k-j)^{3}}\right) \geq \mathcal{F}_{\gamma_{2 k+j}-\gamma_{2 k-j, z}}\left(\frac{1}{10^{3}(2 k-j)^{3}}\right) \geq \alpha .
\end{aligned}
$$

Therefore $\mathcal{F}_{E_{k, j}, z}(1) \geq \alpha$. This shows that $f$ is approximately cubic type mapping. By our assumption, there exists a conditional cubic mapping $C: \mathbb{N} \cup\{0\} \rightarrow X$, such that $\lim _{k \rightarrow \infty} \mathcal{F}_{C(k)-f(k), z}(1)=1$. In particular, $\lim _{k \rightarrow \infty} \mathcal{F}_{C\left(2^{k}\right)-f\left(2^{k}\right), z}(1)=1$. This means that

$$
\lim _{k \rightarrow \infty} \mathcal{F}_{C(1)-y_{2} k, z}\left(\frac{1}{2^{3 k}}\right)=1
$$

Hence the subsequence $\left(y_{2^{k}}\right)$ converges to $y=C(1)$. Therefore, the Cauchy sequence $\left(x_{n}\right)$ also converges to $y$.

\section{Acknowledgements}

The authors would like to thank the anonymous reviewers for their valuable comments.

\section{Authors' contributions}

Both the authors contributed equally and significantly in writing this paper. All authors read and approved the final manuscript.

\section{Competing interests}

The authors declare that they have no competing interests.

Received: 16 December 2011 Accepted: 29 March 2012 Published: 29 March 2012

\section{References}

1. Ulam, SM: A Collection of the Mathematical Problems. Interscience Publ., New York (1960)

2. Hyers, DH: On the stability of the linear functional equation. Proc Nat Acad Sci USA. 27, 222-224 (1941). doi:10.1073/ pnas.27.4.222

3. Aoki, T: On the stability of the linear transformation in Banach spaces. J Math Soc Japan. 2, $64-66$ (1950). doi:10.2969/ jmsj/00210064

4. Rassias, TM: On the stability of the linear mapping in Banach spaces. Proc Am Math Soc. 72, 297-300 (1978). doi:10.1090/S0002-9939-1978-0507327-1 
5. Jun, K-W, Kim, H-M: The generalized Hyers-Ulam-Rassias stability of a cubic functional equation. J Math Anal Appl. 274(2):867-878 (2002). doi:10.1016/S0022-247X(02)00415-8

6. Lee, Y-S, Chung, SY: Stability of the Jensen type functional equation. Banach J Math Anal. 1(1):91-100 (2007)

7. Mirmostafaee, AK, Moslehian, MS: Fuzzy approximately cubic mappings. Infor Sci. 178, 3791-3798 (2008). doi:10.1016/j. ins.2008.05.032

8. Mohiuddine, SA: Stability of Jensen functional equation in intuitionistic fuzzy normed space. Chaos Solitons Fract. 42, 2989-2996 (2009). doi:10.1016/j.chaos.2009.04.040

9. Mohiuddine, SA, Şevli, H: Stability of Pexiderized quadratic functional equation in intuitionistic fuzzy normed space. J Comput Appl Math. 235, 2137-2146 (2011). doi:10.1016/j.cam.2010.10.010

10. Mohiuddine, SA, Cancan, M, Şevli, H: Intuitionistic fuzzy stability of a Jensen functional equation via fixed point technique. Math Comput Model. 54, 2403-2409 (2011). doi:10.1016/j.mcm.2011.05.049

11. Mursaleen, M, Mohiuddine, SA: On stability of a cubic functional equation in intuitionistic fuzzy normed spaces. Chaos, Solitons Fract. 42, 2997-3005 (2009). doi:10.1016/j.chaos.2009.04.041

12. Rassias, TM: On the stability of the quadratic functional equation and its applications. Studia Univ Babeș-Bolyai Math. 43, 89-124 (1998)

13. Menger, K: Statistical metrics. Proc Nat Acad Sci USA. 28, 535-537 (1942). doi:10.1073/pnas.28.12.535

14. Schweizer, B, Sklar, A: Statistical metric spaces. Pacific J Math. 10, 313-334 (1960)

15. Schweizer, B, Sklar, A: Probabilistic Metric Spaces. North Holland, Amsterdam (1983)

16. Goleț, I: On probabilistic 2-normed spaces. Novi Sad J Math. 35, 95-102 (2006)

17. Gähler, S: 2-metrische Räume und ihre topologeische Struktur. Math Nachr. 26, 115-148 (1963). doi:10.1002/ mana.19630260109

18. Mohiuddine, SA, Sevli, H, Cancan, M: Statistical convergence in fuzzy 2-normed space. J Comput Anal Appl. $12(4): 787-798(2010)$

19. Mohiuddine, SA: Some new results on approximation in fuzzy 2-normed spaces. Math Comput Model. 53, 574-580 (2011). doi:10.1016/j.mcm.2010.09.006

20. Mursaleen, M, Alotaibi, A: On 1-Convergence in random 2-normed spaces. Math Slovaco. 61(6):933-940 (2011). doi:10.2478/s12175-011-0059-5

21. Mursaleen, M, Mohiuddine, SA: On ideal convergence in probabilistic normed spaces. Math Slovaca. 62(1):49-62 (2012). doi:10.2478/s12175-011-0071-9

22. Savaş, E, Mohiuddine, SA: $\bar{\lambda}$-statistically convergent double sequences in probabilistic normed spaces. Math Slovaca. 62(1):99-108 (2012). doi:10.2478/s12175-011-0075-5

23. Gürdal, M, Açik, I: On I-Cauchy sequences in 2-normed spaces. Math Inequal Appl. 11(2):349-354 (2008)

24. Gürdal, M, Şahiner, A, Açik, I: Approximation theory in 2-Banach spaces. Nonlinear Anal TMA. 71, 1654-1661 (2009). doi:10.1016/j.na.2009.01.030

25. Şahiner, A, Gürdal, M, Saltan, S, Gunawan, H: Ideal convergence in 2-normed spaces. Taiwanese J Math. 11, 1477-1484 (2007)

26. Mursaleen, M: On statistical convergence in random 2-normed spaces. Acta Sci Math (Szeged). 76, 101-109 (2010)

doi:10.1186/1687-1847-2012-39

Cite this article as: Alotaibi and Mohiuddine: On the stability of a cubic functional equation in random 2-normed spaces. Advances in Difference Equations 2012 2012:39.

\section{Submit your manuscript to a SpringerOpen ${ }^{\circ}$ journal and benefit from:}

- Convenient online submission

- Rigorous peer review

- Immediate publication on acceptance

- Open access: articles freely available online

- High visibility within the field

- Retaining the copyright to your article

Submit your next manuscript at $\boldsymbol{s p r i n g e r o p e n . c o m ~}$ 